\title{
Minkowski's footprint: an ejected common envelope?
}

\author{
Javier Alcolea ${ }^{* \dagger}$ \\ Observatorio Astronómico Nacional (Spain) \\ E-mail: j.alcoleadoan.es
}

Roberto Neri

Institut de Radioastronomie Millimétrique (France)

E-mail: nerieiram.fr

\section{Valentín Bujarrabal}

Observatorio Astronómico Nacional (Spain)

E-mail: v.bujarrabal@oan.es

\begin{abstract}
M 1-92 is a very well studied bipolar pPN that can be considered an archetype of these type of sources: it shows a clear axial symmetry, and the kinematics and momentum excess characteristic of this class of envelopes. We performed sub-arcsecond resolution observations of the $J=2-1$ rotational line of ${ }^{13} \mathrm{CO}$ in $\mathrm{M} 1-92$, using the extended Plateau de Bure interferometer, for better studying the morphology and velocity field of the molecular gas in the central parts of the nebula. We found that the equatorial structure dividing the two lobes is a thin flat disk, which expands radially with a velocity proportional to the distance to the centre. The kinetic age of this equatorial flow is very similar to that found in the two lobes, suggesting that the whole structure was formed as a result of a single event $1200 \mathrm{yr}$ ago. The small widths and velocity dispersion in the gas forming the lobe walls confirm that the acceleration responsible for the nebular shape could not last for more than 100-120 yr. In view of the similarity to the case of $\eta$ Car, we speculate on the possibility that the whole nebula was formed as a result of a magneto-rotational explosion in a common-envelope system. We believe that the role of this formation mechanism, in the in the context of global PNe and pPNe shaping, should be further investigated.
\end{abstract}

From Planets to Dark Energy: the Modern Radio Universe

October 1-5 2007

The University of Manchester, UK

\footnotetext{
* Speaker.

${ }^{\dagger}$ This contribution is based on observations carried out with the IRAM Plateau de Bure Interferometer. IRAM is supported by INSU/CNRS (France), MPG (Germany) and IGN (Spain).
} 


\section{Intruduction}

M 1-92, also known as Minkowski's footprint, is a bipolar pre-planetary nebula (pPN). The central object is a binary system consisting of a 6,500 K primary star and a $18,000 \mathrm{~K}$ secondary [2]. A distance of $2.5 \mathrm{kpc}$ is assumed adopting a normal post-AGB luminosity of $10^{4} L_{\odot}$ [5]. This axissymmetric nebula has a size of $11^{\prime \prime}$ in the axial direction and $6^{\prime \prime}$ in the equatorial one, consisting of a two-lobe reflection nebula, divided by a dense equatorial component. The axis of symmetry is oriented at a position angle of $311^{\circ}$, and it is inclined with respect to the plane of the sky by $35^{\circ}$, with the northwest lobe pointing to us. Optical spectroscopy shows the presence of flows of ionised gas close to the star, with expansion velocities up to $750 \mathrm{~km} \mathrm{~s}^{-1}$ [2]. Line emission in $\mathrm{H} \alpha$ and in OI, OIII, NII, \& SII is detected from the middle of the two lobes, tracing the location of shocks propagating along these jets [4]. The mass of the ionised gas is very low, $10^{-3} M_{\odot}$, compared with the total mass of the nebula, $0.9 M_{\odot}$ [4], which is still largely in the form of molecular gas [3].

\section{Observations \& results}

Using the new extended configurations of the IRAM Plataeu de Bure Interferometer (PdBI), we conducted additional observations of the $J=2-1$ rotational line of ${ }^{13} \mathrm{CO}$ at $220 \mathrm{GHz}$ in $\mathrm{M} 1-92$. We obtained new data in the A6q \& B6q antenna layouts in the winter of 2006. After calibration, map production and cleaning we found that $30 \%-70 \%$ of the flux was lost because of the lack of short baselines in the new data. Since the new data set was obtained with the same spectral setup as before, we merged these data with those from our previous observations [3] (see [1] for additional details), resulting in maps with no significant missing flux, but with sub-arcsecond spatial resolution: beam size of 0 '. $50 \times 0$ '! 35 . We also produced higher detailed maps using just the most extended configuration. Although these maps have lower $\mathrm{S} / \mathrm{N}$ and most of the flux is lost, they are very useful to investigate the structure and dynamics of the equatorial disk dividing the two lobes.

The results (see figure) clearly display the structure and kinematics of the molecular gas in M 1-92. The two emptied lobes and the dividing equatorial structure are obvious, as well as the prominent linear velocity gradient in the axial directions: the Hubble-like velocity field often found in pPNe. It is noteworthy the small width of the walls of the lobes, less than $10^{16} \mathrm{~cm}$, and their low excitation $\left(T_{\mathrm{Br}}\right.$ about $\left.10 \mathrm{~K}\right)$. However, most surprising is that the velocity field seen in the equatorial region, i.e. the disk, is not compatible with a relic of an AGB envelope. We do not see the signatures of an expansion with a constant radial velocity. On the contrary it seems that the velocity also increases linearly with the distance in this equatorial plane too. We derive a kinetic life-time of $1370 \mathrm{yr}$ for the disk, to be compared with the $1060 \mathrm{yr}$ found for the bipolar axial flow. In fact, adopting an inclination angle of the symmetry axis of $38^{\circ} .5$ (still compatible with all the data), both ages become the same, $1200 \mathrm{yr}$. Using the higher resolution data, we confirm these results for the disk, that is flat and $1^{\prime \prime} 4$ in diameter.

\section{On the nature of M1-92}

If we assume that a single Hubble-like velocity field applies to all parts of the nebula, the easiest explanation for this kinematics would be that M 1-92 resulted from a single acceleration 


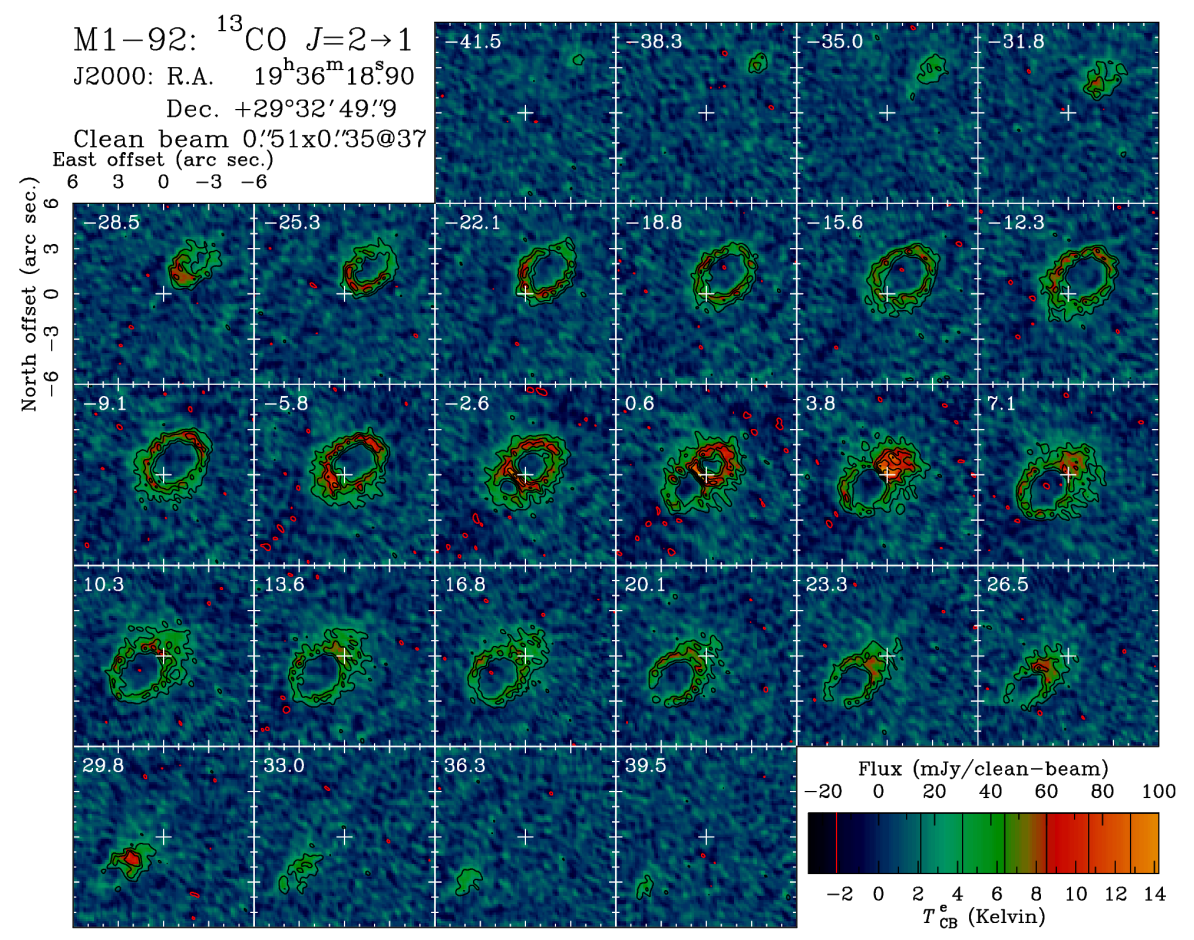

Figure 1: Fig 3. The $3.25 \mathrm{~km} \mathrm{~s}^{-1}$ spectral resolution channel maps of ${ }^{13} \mathrm{CO} J=2-1$ in $\mathrm{M} 1-92$ obtained from the old+new data combined.

event. Given the small width and velocity dispersion of the lobe walls, that interaction lasted less than about $10 \%$ of the kinetic age of the gas, i.e. less than $120 \mathrm{yr}$, resulting in the astonishing mass loss rate of at least $7.510^{-3} M_{\odot} \mathrm{yr}^{-1}$. We see no traces of the former AGB envelope, just the result of this huge post-AGB explosion. The above depicted scenario resembles a 1-2 orders of magnitude scale-down version of the $\eta$ Car nebula. For this unique object, a model has been developed in which a magneto-rotational explosion can launch flows in the axial and equatorial directions [6]. For this mechanism to operate, it is necessary to have a binary system in a common envelope phase [7], a scenario that we believe should be further investigated for the case of M 1-92. If true, the nebula will be just the result of the explosive ejection of the former common envelope of the binary system.

\section{References}

[1] J. Alcolea, R. Neri, V. Bujarrabal, (2007), A\&A 468, L41

[2] A. Arrieta, S. Torres-Peimbert, L. Georgiev, (2005), ApJ 623, 252

[3] V. Bujarrabal, J. Alcolea, R. Neri, (1998), ApJ 504, 915

[4] V. Bujarrabal, J. Alcolea, R. Sahai, J. Zamorano, A.A. Zijlstra, (1998), A\&A 331, 361

[5] M. Cohen, L.V. Kuhi, (1977), ApJ 213, 79

[6] S. Matt, A. Frank, E.G. Blackman, (2006), ApJ 647, L45

[7] J. Nordhaus, E.G. Blackman, (2006), MNRAS 370, 2004 